\title{
HETEROCLINICS FOR NON AUTONOMOUS THIRD ORDER DIFFERENTIAL EQUATIONS
}

\author{
Denis Bonheure - José Ángel Cid \\ Colette De Coster - Luis Sanchez
}

AbStRact. We study the existence of heteroclinics connecting the two equilibria \pm 1 of the third order differential equation

$$
u^{\prime \prime \prime}=f(u)+p(t) u^{\prime}
$$

where $f$ is a continuous function such that $f(u)\left(u^{2}-1\right)>0$ if $u \neq \pm 1$ and $p$ is a bounded non negative function. Uniqueness is also addressed.

\section{Introduction}

The existence of kink solutions or heteroclinic orbits for the third order problem

$$
u^{\prime \prime \prime}=f(u), \quad u(-\infty)=u_{-}, \quad u(+\infty)=u_{+},
$$

2010 Mathematics Subject Classification. 34B40, 34C37.

Key words and phrases. Third order, heteroclinic, boundary value problems in unbounded intervals.

The second named author partially supported by Ministerio de Educación y Ciencia, Spain, Project MTM2010-15314 and FEDER.

The fourth named author supported by Fundação para a Ciência e a Tecnologia, Financiamento Base ISL-209 (2010) and PEst-OE/MAT/UI0209/2011. 
arises for instance in the study of regularization of the Cauchy problem for onedimensional hyperbolic conservation laws

$$
u_{t}+g(u)_{x}=0, \quad u(0, x)=\bar{u}(x) .
$$

It is known that the single shock wave joining the two states $u_{-}$(on the left) and $u_{+}$(on the right)

$$
u(t, x):= \begin{cases}u_{-} & \text {for } x<\lambda t, \\ u_{+} & \text {for } x>\lambda t,\end{cases}
$$

is a weak solution of (1.2) if and only if its speed $\lambda$ satisfies the Rankine-Hugoniot equation (see [1, Lemma 4.2])

$$
g\left(u_{+}\right)-g\left(u_{-}\right)=\lambda\left(u_{+}-u_{-}\right) .
$$

However weak solutions of (1.2) are in general not unique. A way to regularize problem (1.2) is to search for weak solutions which are limits as $\varepsilon \rightarrow 0^{+}$of solutions of

$$
u_{t}^{\varepsilon}+g\left(u^{\varepsilon}\right)_{x}=\varepsilon A\left(u^{\varepsilon}\right), \quad u^{\varepsilon}(0, x)=\bar{u}(x),
$$

where $A$ is a differential operator of higher order in $x$ (the viscosity). A choice of $A$ is admissible, in the sense of Gelfand [4], if shock wave solutions given by (1.3) can be obtained as limits of solutions of (1.4). When $A$ is a perfect derivative the admissibility is equivalent to the existence of a heteroclinic connection between $u_{-}$and $u_{+}$for an autonomous equation. In particular, the question of the admissibility of operator $A(u)=-u_{x x x x}$ leads to problem (1.1) (see [8], [9]).

In this work we are mainly motivated by the non autonomous version of a related problem studied in [6], [12]

$$
u^{\prime \prime \prime}=f(u)+p(t) u^{\prime}, \quad u(-\infty)=-1, \quad u(+\infty)=1,
$$

where $f: \mathbb{R} \rightarrow \mathbb{R}$ and $p: \mathbb{R} \rightarrow \mathbb{R}$ satisfy the following assumptions:

(f1) $f: \mathbb{R} \rightarrow \mathbb{R}$ is continuous and such that $f(-1)=f(1)=0$;

(p) $p$ is continuous and there exists $M>0$ such that, for all $t \in \mathbb{R}, 0 \leq$ $p(t) \leq M$.

Clearly, under these assumptions, $u=-1$ and $u=1$ are constant solutions of the equation (1.5), so that we are looking for a heteroclinic connection between these equilibria.

Our main example is $f(u)=u^{2}-1$ and $p$ constant, which satisfies the above conditions as well as

(s) $f$ is even,

(s') $p$ is even, 
and $f$ is increasing on $[0,+\infty[$. This last assumption is too strong for most of our aims. In our results we shall consider the following assumptions on $f$ and

$$
F(s)=\int_{0}^{s} f(r) d r .
$$

(h1) There exists $N_{0}>1$ such that

$$
\forall u \in\left[0, N_{0}\right] \backslash\{1\}, \quad f(u)(u-1)>0 \quad \text { and } \quad F\left(N_{0}\right) \geq 0 ;
$$

(h2) There exist $\alpha<-1$ and $\beta>1$ such that,

$$
\begin{gathered}
\forall u \in[\alpha, \beta] \backslash\{-1,1\}, \quad f(u)\left(u^{2}-1\right)>0, \\
F(\beta)=F(-1) \quad \text { and } \quad F(\alpha)=F(1) ;
\end{gathered}
$$

(h3) $f$ satisfies (h2), is nondecreasing on $[0, \beta]$ and nonincreasing on $[\alpha, 0]$;

(h4) $f$ satisfies (h2) together with

$$
\int_{\alpha}^{0} F(s) d s>0 \text { and } \int_{0}^{\beta} F(s) d s<0 .
$$

Remark 1.1. Note that (h2) implies (h1) with $N_{0}=\beta$. On the other hand (h1) is more general than (h2). Indeed, if $f$ satisfies (f1), $f(u)\left(u^{2}-1\right)>0$ for all $u \neq \pm 1$, and

$$
0<-\int_{0}^{1} f(u) d u<\int_{1}^{\infty} f(u) d u \leq-\int_{-1}^{1} f(u) d u,
$$

then $f$ satisfies (h1) but not (h2).

Observe also that in case $f$ is continuous on $\mathbb{R}$, nondecreasing on $\mathbb{R}^{+}$, nonincreasing on $\mathbb{R}^{-}$and such that, for all $u \neq \pm 1, f(u)\left(u^{2}-1\right)>0$, we have that $F(+\infty)=+\infty$ and $F(-\infty)=-\infty$ and hence (h2) is satisfied.

In comparison with second order (and fourth order) equations with monostable or bistable nonlinearities which have been extensively studied through variational or topological arguments, see for instance [2], third order equations have been much less considered. Problem (1.5) however already received attention in the literature. Solvability of (1.5) with $f(u)=u^{2}-1$ and $p \equiv 0$ was given independently by Kopell and Howard [5] and by Conley [3] (see also [11, p. 456]). For general $f$ there are several results due to Mock for $p \equiv 0[8]$, [9] and Manukian and Schecter [6, Theorem 5.2] for $p \equiv \beta>0$. Uniqueness of the connecting orbit for $f(u)=u^{2}-1$ was proved by McCord [7] and later by Toland [12]. Our results will complement and improve some of the previous ones.

This paper is organized as follows: in Section 2 we prove that, under assumptions (f1), (p) and $f(u)\left(u^{2}-1\right)>0$ in a suitable interval except \pm 1 , the 
existence of a solution of (1.5) is equivalent to the existence of a bounded non constant solution of

$$
u^{\prime \prime \prime}=f(u)+p(t) u^{\prime}
$$

In Section 3, we prove the existence of such a solution in case $f$ and $p$ satisfy (f1), (h1), (p), (s) and ( $\left.\mathrm{s}^{\prime}\right)$ and hence also the existence of a solution of (1.5), while in Section 4, we obtain the existence of a solution of (1.5) under assumptions (f1), (p) and (h2). We do not need a Lipschitz condition as in the above quoted references since we use a different approach based on degree theory combined with an approximation procedure.

In Section 5 we prove, among other things, that, in addition to (f1), (h3), (h4), it is sufficient to assume $f$ is locally Lipschitz on $\mathbb{R}$ and $p$ is a non negative constant in order to get uniqueness for the solution of (1.5).

\section{Bounded solutions versus heteroclinics}

We start with an analysis of the behaviour of bounded solutions at infinity.

Proposition 2.1. Assume the conditions (f1), (p) and $f$ has only isolated zeros.

(a) If $u$ is a solution of (1.6) in $\mathbb{R}$, bounded together with $p u^{\prime}$, then, for $i \in\{1,2,3\}, u^{(i)}( \pm \infty)=0, u(+\infty)=a^{+}$and $u(-\infty)=a^{-}$with $f\left(a^{ \pm}\right)=0$.

(b) If in addition $u$ is non constant and for all $x \in\left[-\|u\|_{\infty},\|u\|_{\infty}\right] \backslash\{ \pm 1\}$, $f(x)\left(x^{2}-1\right)>0$, then $u(-\infty)=-1$ and $u(+\infty)=1$.

Proof. First observe that, as $u$ and $p u^{\prime}$ are bounded, by the equation satisfied by $u$, we have $u^{\prime \prime \prime}$ bounded on $\mathbb{R}$ and hence, by interpolation, $u^{\prime \prime}$ and $u^{\prime}$ are bounded too.

Claim 1. $u^{\prime \prime}(+\infty)=0$. Multiplying the equation $u^{\prime \prime \prime}=f(u)+p(t) u^{\prime}$ by $u^{\prime}$ and integrating on $[0, t]$ we obtain

$$
\begin{aligned}
u^{\prime}(t) u^{\prime \prime}(t)-u^{\prime}(0) u^{\prime \prime}(0)-F(u(t))+ & F(u(0)) \\
& =\int_{0}^{t} u^{\prime \prime 2}(s) d s+\int_{0}^{t} p(s) u^{\prime 2}(s) d s .
\end{aligned}
$$

By hypothesis, the left hand side is bounded in $\mathbb{R}$ and therefore

$$
\int_{0}^{\infty} p(s) u^{\prime 2}(s) d s+\int_{0}^{\infty} u^{\prime \prime 2}(s) d s<\infty .
$$

Since $p$ is nonnegative, we infer from the square integrability of $u^{\prime \prime}$ and the boundedness of $u^{\prime \prime \prime}$ that $u^{\prime \prime}(+\infty)=0$.

Claim 2. $u^{\prime}(+\infty)=0$. First it is clear that $u^{\prime}$ cannot accumulate to a positive or negative value. If $u^{\prime}$ has more than a cluster value, then $\mid u^{\prime}(x)-$ 
$u^{\prime}(y) \mid \geq \varepsilon>0$ implies $|x-y| \rightarrow \infty$ because $u^{\prime \prime}(+\infty)=0$. Then it is easy to reach a contradiction using the boundedness of $u$.

Claim 3. $u^{\prime \prime \prime}(+\infty)=0$ and $u(+\infty)=a^{+}$with $f\left(a^{+}\right)=0$. Equation $(2.1)$ together with (2.2), Claims 1 and 2 imply that $F(u(+\infty))$ exists. As $F$ is not constant in any interval, it follows that $u(+\infty)=a^{+}$exists. Going back to the equation (1.6) we conclude that $u^{\prime \prime \prime}(+\infty)=f\left(a^{+}\right)$. Since $u^{\prime \prime}$ is bounded we obtain $f\left(a^{+}\right)=0$.

ClaIm 4. For $i \in\{1,2,3\}, u^{(i)}(-\infty)=0$ and $u(-\infty)=a^{-}$with $f\left(a^{-}\right)=0$. The proof is the same as in the previous Claims.

Claim 5. In case $u$ is not constant and, for all $x \in\left[-\|u\|_{\infty},\|u\|_{\infty}\right] \backslash\{ \pm 1\}$, $f(x)\left(x^{2}-1\right)>0$, then $u(-\infty)=-1$ and $u(+\infty)=1$. Observe that, by assumption, $\left\{a^{+}, a^{-}\right\} \subset\{-1,1\}$. Moreover, along the solutions of (1.6), we have $\left(F(u(t))-u^{\prime \prime}(t) u^{\prime}(t)\right)^{\prime}=-p(t) u^{\prime 2}(t)-u^{\prime \prime 2}(t)$ from which we deduce that $F(u(t))-u^{\prime \prime}(t) u^{\prime}(t)$ is nonincreasing and in fact decreasing in case $u^{\prime \prime}(t) \neq 0$. Hence, as $u$ is not constant, we have

$$
\begin{aligned}
F(u(+\infty)) & =\lim _{t \rightarrow+\infty}\left(F(u(t))-u^{\prime \prime}(t) u^{\prime}(t)\right) \\
& <\lim _{t \rightarrow-\infty}\left(F(u(t))-u^{\prime \prime}(t) u^{\prime}(t)\right)=F(u(-\infty)) .
\end{aligned}
$$

The result follows from $\{u(+\infty), u(-\infty)\} \subset\{-1,1\}$ and $F(1)<F(-1)$.

Remark 2.2. Observe that Proposition 2.1 implies that, under the assumptions (f1), (p) and if, for all $x \neq \pm 1, f(x)\left(x^{2}-1\right)>0$, the problem

$$
u^{\prime \prime \prime}=f(u)+p(t) u^{\prime}, \quad u(-\infty)=1, \quad u(+\infty)=-1,
$$

has no $\mathcal{C}^{1}$-bounded solution.

Define the space $\mathcal{C B}^{3}(\mathbb{R})=\left\{u \in \mathcal{C}^{3}(\mathbb{R}) \mid u, u^{\prime}, u^{\prime \prime}, u^{\prime \prime \prime} \in L^{\infty}(\mathbb{R})\right\}$.

Proposition 2.3. Under the assumptions (f1), (p) and (h2), any solution $u \in \mathcal{C B}^{3}(\mathbb{R})$ of $(1.5)$ takes values in $[\alpha, \beta]$.

Proof. Recall first that $F(u(t))-u^{\prime \prime}(t) u^{\prime}(t)$ is nonincreasing along the solutions of (1.5). Using Proposition 2.1, we have for all $t \in \mathbb{R}$,

$$
\begin{aligned}
F(1) & =\lim _{t \rightarrow+\infty}\left(F(u(t))-u^{\prime \prime}(t) u^{\prime}(t)\right) \\
& \leq F(u(t))-u^{\prime \prime}(t) u^{\prime}(t) \leq \lim _{t \rightarrow-\infty}\left(F(u(t))-u^{\prime \prime}(t) u^{\prime}(t)\right)=F(-1) .
\end{aligned}
$$

Hence, for every critical value $\bar{t}$ of $u$ we have

$$
F(1) \leq F(u(\bar{t})) \leq F(-1) .
$$

The result then follows from the fact that $u(+\infty)=1$ and $u(-\infty)=-1$. 
REMARK 2.4. Suppose that in addition to (f1), there exists $c>0$ such that $f(u)(u-1) \geq c(u-1)^{2}$ for all $u>0$ (this is in particular true for $f(u)=u^{2}-1$ ). Then it is easy to see that any bounded solution of $u^{\prime \prime \prime}=f(u)$ belongs to an affine translate of the space $H^{2}(\mathbb{R})$ (or we can write $u \mp 1 \in H^{2}\left(\mathbb{R}^{ \pm}\right)$). In fact multiplying the equation by $u-1$ and integrating in $[0, T]$ we see that the integral $\int_{0}^{T} f(u(s))(u(s)-1) d s$ is bounded independently of $T>0$. This implies that $\int_{0}^{\infty} f(u(s))(u(s)-1) d s$ exists and by the above condition

$$
\int_{0}^{\infty}(u(s)-1)^{2} d s<\infty .
$$

As $\int_{0}^{\infty} u^{\prime \prime 2}(s) d s<\infty$ the conclusion follows from standard interpolation.

\section{A boundary value problem in the half-line:}

\section{bounded solutions yielding odd heteroclinics under symmetry}

To solve (1.5) in case $f$ and $p$ are even it is enough to find a solution of the boundary value problem

$$
u^{\prime \prime \prime}=f(u)+p(t) u^{\prime}, \quad u(0)=u^{\prime \prime}(0)=0, \quad u(+\infty)=1 .
$$

Indeed, if $u$ is a solution of (3.1) then the odd extension of $u$ solves (1.5).

To solve (3.1), using Proposition 2.1, we consider the approximated problem in a finite interval $[0, n], n \in \mathbb{N}$,

$$
u^{\prime \prime \prime}=f(u)+p(t) u^{\prime}, \quad u(0)=u^{\prime \prime}(0)=0, \quad u^{\prime}(n)=0 .
$$

Lemma 3.1. Suppose that $f: \mathbb{R} \rightarrow \mathbb{R}$ and $p: \mathbb{R} \rightarrow \mathbb{R}$ satisfy conditions (f1), (p), (h1). Then for each $n \in \mathbb{N}$, there exists a solution $u_{n}$ of (3.2) with $0 \leq u_{n} \leq$ $N_{0}$ on $[0, n]$, where $N_{0}$ is given by (h1).

ProOF. We divide the proof into several steps.

Step 1. The modified problem. We define the function $f^{*}: \mathbb{R} \rightarrow \mathbb{R}$ as

$$
f^{*}(u)= \begin{cases}f\left(N_{0}\right), & \text { if } u>N_{0}, \\ f(u), & \text { if } u \in\left[0, N_{0}\right], \\ f(0), & \text { if } u<0,\end{cases}
$$

and consider the modified problem

$$
u^{\prime \prime \prime}=f^{*}(u)+p(t) u^{\prime}, \quad u(0)=u^{\prime \prime}(0)=0, \quad u^{\prime}(n)=0 .
$$

Step 2. Reduction to a fixed point problem.

Claim 1. For each $h \in \mathcal{C}([0, n])$, the linear problem

$$
u^{\prime \prime \prime}-p(t) u^{\prime}=h(t), \quad u(0)=u^{\prime \prime}(0)=0, \quad u^{\prime}(n)=0,
$$

has a unique solution. 
As is well known, it is sufficient to prove that the problem

$$
u^{\prime \prime \prime}-p(t) u^{\prime}=0, \quad u(0)=u^{\prime \prime}(0)=0, \quad u^{\prime}(n)=0,
$$

has only the trivial solution. In fact, if (3.5) has a nontrivial solution, let $v=u^{\prime}$. Then $v$ satisfies

$$
v^{\prime \prime}-p(t) v=0, \quad v^{\prime}(0)=0, \quad v(n)=0 .
$$

Multiplying the equation by $v$ and integrating, we have

$$
\int_{0}^{n}\left(v^{\prime 2}(t)+p(t) v^{2}(t)\right) d t=0 .
$$

This implies that $v^{\prime} \equiv 0$ and as $v(n)=0$ we obtain $v \equiv 0$, i.e. $u^{\prime} \equiv 0$. Since $u(0)=0$, it follows that $u \equiv 0$.

By the above claim, we can define the solution operator $K: \mathcal{C}([0, n]) \rightarrow$ $\mathcal{C}([0, n])$ corresponding to $(3.4)$. Then let $S: \mathcal{C}([0, n]) \rightarrow \mathcal{C}([0, n])$ be given by

$$
S u=K\left(f^{*}(u)\right) .
$$

It is clear that $S$ is a completely continuous operator and that $u$ is a solution of (3.3) if and only if $u$ is a fixed point of $S$. In order to obtain a fixed point we consider the homotopy

$$
u=K\left(\lambda f^{*}(u)\right), \quad \lambda \in[0,1],
$$

which is equivalent to the problem

$$
u^{\prime \prime \prime}=\lambda f^{*}(u)+p(t) u^{\prime}, \quad u(0)=u^{\prime \prime}(0)=0, \quad u^{\prime}(n)=0 .
$$

Step 3. A priori estimates.

Claim 2. For all $\lambda \in[0,1]$, any solution of $(3.7)$ is nonnegative on $[0, n]$.

Let $u$ be a solution of (3.7).

Case 1. $\lambda=0$. As in Step 2 above, we know that the solution of

$$
u^{\prime \prime \prime}=p(t) u^{\prime}, \quad u(0)=u^{\prime \prime}(0)=0, \quad u^{\prime}(n)=0 .
$$

is $u \equiv 0$.

Case 2. $\lambda \in] 0,1]$. Assume by contradiction that $u$ takes negative values. Then the boundary conditions imply that for some $t_{1}<t_{2}$ we have $u\left(t_{1}\right)=0$, and $u(t)<0$ for all $t \in] t_{1}, t_{2}\left[, u^{\prime}\left(t_{2}\right)=0\right.$ and for all $\left.t \in\right] t_{1}, t_{2}\left[, u^{\prime}(t)<0\right.$. Otherwise $t_{1}$ would be an accumulation point of critical points of $u$, implying $0=u^{\prime \prime \prime}\left(t_{1}\right)-p\left(t_{1}\right) u^{\prime}\left(t_{1}\right)=\lambda f(0)<0$, a contradiction. Now we have, for some $t \in] t_{1}, t_{2}[$,

$$
0=u^{\prime}\left(t_{2}\right)=u^{\prime}\left(t_{1}\right)+u^{\prime \prime}\left(t_{1}\right)\left(t_{2}-t_{1}\right)+\left[\lambda f^{*}(u(t))+p(t) u^{\prime}(t)\right] \frac{\left(t_{2}-t_{1}\right)^{2}}{2} .
$$


Since $\lambda f^{*}(u(t))+p(t) u^{\prime}(t) \leq \lambda f(0)<0$ and $u^{\prime}\left(t_{1}\right) \leq 0$ then $u^{\prime \prime}\left(t_{1}\right)>0$ and hence $t_{1}>0$. Multiplying the equation (3.7) by $u^{\prime}$ and integrating by parts between 0 and $t_{1}$ we obtain the contradiction

$$
\begin{aligned}
0 & >u^{\prime}\left(t_{1}\right) u^{\prime \prime}\left(t_{1}\right)-\int_{0}^{t_{1}} u^{\prime \prime 2}(s) d s \\
& =\int_{0}^{t_{1}} \lambda f^{*}(u(s)) u^{\prime}(s) d s+\int_{0}^{t_{1}} p(s) u^{\prime 2}(s) d s=\int_{0}^{t_{1}} p(s) u^{\prime 2}(s) d s \geq 0 .
\end{aligned}
$$

Claim 3. For any $n \in \mathbb{N}, \lambda \in[0,1]$ and any solution $u$ of (3.7) we have,

$$
|u(t)| \leq N_{0}, \quad \text { for all } t \in[0, n], .
$$

By Claim 2 we have that $u(t) \geq 0$ for all $t \in[0, n]$. Let a solution $u$ of (3.7) attain a positive maximum at some point $\left.\left.t_{0} \in\right] 0, n\right]$. This implies in particular that $\lambda \neq 0$. Now, multiplying (3.7) by $u^{\prime}$ and integrating in $\left[0, t_{0}\right]$, we have

$$
0 \geq-\int_{0}^{t_{0}} u^{\prime \prime 2}(s) d s-\int_{0}^{t_{0}} p(s) u^{\prime 2}(s) d s=\lambda F^{*}\left(u\left(t_{0}\right)\right),
$$

with $F^{*}(u)=\int_{0}^{u} f^{*}(s) d s$. Hence by assumption (h1) and construction, we obtain that $0 \leq u(t) \leq N_{0}$.

Step 4. Conclusion. By standard results of Leray-Schauder degree theory the equation (3.6) has a solution $u$ for $\lambda=1$ which is a solution of (3.3). Moreover by Claims 2 and 3 we have that $0 \leq u \leq N_{0}$ and hence it is also a solution of (3.2).

REMARK 3.2. We do not use the all strength of (h1). We just used the fact that $f(0)<0$ and there exists $N_{0}>0$ such that $F\left(N_{0}\right) \geq 0$. In that case, without loss of generality, we can assume $f\left(N_{0}\right) \geq 0$.

LEMma 3.3. Under the assumptions of Lemma 3.1, there exists a number $K>0$ with the property that, for all $n \in \mathbb{N}$,

$$
\left\|u_{n}\right\|_{\mathcal{C}^{3}([0, n])} \leq K
$$

Proof. We first show that $\left\|u_{n}^{\prime \prime}\right\|_{L^{2}(0, n)}$ is bounded independently of $n$. Indeed, multiplying the equation in (3.2) by $u_{n}^{\prime}$ and integrating by parts between 0 and $n$, using the boundary conditions we obtain

$$
\int_{0}^{n} u_{n}^{\prime \prime 2}(s) d s=-\int_{0}^{n} f\left(u_{n}(s)\right) u_{n}^{\prime}(s) d s-\int_{0}^{n} p(s) u_{n}^{\prime 2}(s) d s \leq-\min _{\left[0, N_{0}\right]} F .
$$

Let us extend $u_{n}$ to $\left[0,+\infty\left[\right.\right.$ with the constant value $u_{n}(n)$ in $[n,+\infty[$, and define $v_{n}$ as the odd extension of $u_{n}$ to $\mathbb{R}$. Then $v_{n} \in \mathcal{C}^{1}(\mathbb{R})$ and by the GagliardoNirenberg's interpolation inequality [10], there is a constant $C$ such that

$$
\left\|v_{n}^{\prime}\right\|_{\mathcal{C}(\mathbb{R})} \leq C\left\|v_{n}^{\prime \prime}\right\|_{L^{2}(\mathbb{R})}^{2 / 3}\left\|v_{n}\right\|_{\mathcal{C}(\mathbb{R})}^{1 / 3} .
$$


Since

$$
\left\|v_{n}^{\prime}\right\|_{\mathcal{C}(\mathbb{R})}=\left\|u_{n}^{\prime}\right\|_{\mathcal{C}([0, n])}, \quad\left\|v_{n}^{\prime \prime}\right\|_{L^{2}(\mathbb{R})}=2\left\|u_{n}^{\prime \prime}\right\|_{L^{2}(0, n)}, \quad\left\|v_{n}\right\|_{\mathcal{C}(\mathbb{R})}=\left\|u_{n}\right\|_{\mathcal{C}([0, n])},
$$

we infer

$$
\sup _{n}\left\|u_{n}^{\prime}\right\|_{\mathcal{C}([0, n])}<\infty
$$

and the differential equation yields

$$
\sup _{n}\left\|u_{n}^{\prime \prime \prime}\right\|_{\mathcal{C}([0, n])}<\infty .
$$

The conclusion now follows from standard interpolation.

Proposition 3.4. Assume hypotheses (f1), (p), (h1). Then the boundary value problem (3.1) has a solution $u \in \mathcal{C}^{3}([0,+\infty[)$ which is nonnegative on $\left[0,+\infty\left[\right.\right.$ and such that $u^{\prime}, u^{\prime \prime}$ and $u^{\prime \prime \prime}$ are bounded in $\mathbb{R}^{+}$.

Proof. By Lemmas 3.1 and 3.3 we have that, for each $n \in \mathbb{N}$, the equation $u^{\prime \prime \prime}=f(u)+p(t) u^{\prime}$ has a solution $u_{n}$ defined in $[0, n], u_{n}(0)=u_{n}^{\prime \prime}(0)=0$, $0 \leq u_{n} \leq N_{0}$ and $u_{n}^{\prime}$, $u_{n}^{\prime \prime}$ and $u_{n}^{\prime \prime \prime}$ are bounded by a constant $M>0$ which is independent of $n \in \mathbb{N}$. Then using Ascoli's theorem and the Cantor diagonal process we can select a sequence of values of $n_{k} \rightarrow \infty$ and $u \in \mathcal{C}^{3}([0,+\infty[)$ so that for any $a>0$ we have that $u_{n_{k}}$ converges to $u$ in $\mathcal{C}^{3}([0, a])$ and $u$ solves $u^{\prime \prime \prime}=f(u)+p(t) u^{\prime}$ and satisfies the boundary conditions at $t=0$. As $u(0)=0$, $u$ is not a constant solution and by the arguments in the proof of Proposition 2.1, $u$ satisfies the boundary condition at infinity as well.

Extending any solution of (3.1) by oddness, the last proposition implies:

Theorem 3.5. Assume that hypotheses (f1), (p), (h1), (s) and (s') hold. Then (1.5) has an odd solution $u \in \mathcal{C B}^{3}(\mathbb{R})$ which nonnegative in $] 0,+\infty[$ and satisfies

$$
u^{\prime}( \pm \infty)=u^{\prime \prime}( \pm \infty)=u^{\prime \prime \prime}( \pm \infty)=0 .
$$

REMARK 3.6. The function $F(u)-u^{\prime} u^{\prime \prime}$ plays the role of a Liapunov function for the equation $u^{\prime \prime \prime}=f(u)+p(t) u^{\prime}$. In fact in the case where $p$ is constant and we have uniqueness of the Cauchy problem, for instance when $f$ is locally Lipschitzcontinuous, we can obtain a proof of the Theorem 3.5 using that the Liapunov function is strictly decreasing along the nonconstant solutions and the La Salle invariance principle [13].

Moreover, the existence of the Liapunov function may be used to see that the problem

$$
u^{\prime \prime \prime}=f(u), \quad u(-\infty)=-1, \quad u(+\infty)=1,
$$

has no solution if $f$ is a continuous odd function with $f(1)=0$. 


\section{The non-symmetric problem}

If we drop the assumptions of symmetry (s) and ( $\left.\mathrm{s}^{\prime}\right)$ the existence of heteroclinics of (1.5) becomes considerably more complicated.

Theorem 4.1. Assume that hypotheses (f1), (p) and (h2) hold. Then (1.5) has a solution $u \in \mathcal{C B}^{3}(\mathbb{R})$ which satisfies

$$
u^{\prime}( \pm \infty)=u^{\prime \prime}( \pm \infty)=u^{\prime \prime \prime}( \pm \infty)=0 .
$$

Proof. As in the symmetric case, to solve (1.5) we start with an approximated problem in a finite interval $[-n, n], n \in \mathbb{N}$,

$$
u^{\prime \prime \prime}=f(u)+p(t) u^{\prime}, \quad u^{\prime}(-n)=0, \quad u(0)=0, \quad u^{\prime}(n)=0 .
$$

and we prove the equivalent of Lemma 3.1 for this problem, i.e. for all $n \in \mathbb{N}$, there exists a solution $u_{n}$ of (4.1) with, for all $t \in[-n, n], \alpha<u(t)<\beta$ where $\alpha$ and $\beta$ are given by (h2).

We divide the proof into several steps.

Step 1. The modified problem. We define the functions $f_{+}, f_{-}:[-n, n] \times \mathbb{R}$ $\rightarrow \mathbb{R}$ by

$$
f_{+}(u)=\left\{\begin{array}{ll}
f(\beta) & \text { if } u>\beta, \\
f(u) & \text { if } u \in[-1, \beta], \\
0 & \text { if } u<-1,
\end{array} \quad f_{-}(u)= \begin{cases}0 & \text { if } u>1, \\
f(u) & \text { if } u \in[\alpha, 1] \\
f(\alpha) & \text { if } u<\alpha .\end{cases}\right.
$$

and we set

Consider then the modified problem

$$
f^{*}(t, u)= \begin{cases}f_{+}(u) & \text { if } t \geq 0 \\ f_{-}(u) & \text { if } t<0\end{cases}
$$

$$
u^{\prime \prime \prime}=f^{*}(t, u)+p(t) u^{\prime}, \quad u^{\prime}(-n)=0, \quad u(0)=0, \quad u^{\prime}(n)=0 .
$$

Step 2. Reduction to a fixed point problem.

Claim 1. For each $h \in \mathcal{C}([-n, n])$, the linear problem

$$
u^{\prime \prime \prime}-p(t) u^{\prime}=h(t), \quad u^{\prime}(-n)=0, \quad u(0)=0, \quad u^{\prime}(n)=0,
$$

has a unique solution.

The proof follows as in Lemma 3.1.

By the above claim, we can define the solution operator $K: \mathcal{C}([-n, n]) \rightarrow$ $\mathcal{C}([-n, n])$ corresponding to (4.3). Let

$$
\begin{array}{r}
\Omega=\{u \in \mathcal{C}([-n, n]) \mid u(-n)<1 \text { and } u(n)>-1 \\
\text { and, for all } t \in[-n, n], \alpha<u(t)<\beta\} .
\end{array}
$$


Then let $S: \bar{\Omega} \rightarrow \mathcal{C}([-n, n])$ be given by

$$
S u=K\left(f^{*}(t, u)\right) .
$$

It is clear that $S$ is a completely continuous operator and that $u$ is a solution of (4.2) if and only if $u$ is a fixed point of $S$. In order to obtain a fixed point we consider the homotopy

$$
u=K\left(\lambda f^{*}(t, u)\right), \quad \lambda \in[0,1],
$$

which is equivalent to the problem

$$
u^{\prime \prime \prime}=\lambda f^{*}(t, u)+p(t) u^{\prime}, \quad u^{\prime}(-n)=0, \quad u(0)=0, \quad u^{\prime}(n)=0 .
$$

Step 3. A priori estimates. Let us prove that, for all $\lambda \in[0,1]$, there is no solution of (4.5) on $\partial \Omega$.

Claim 2. For $\lambda=0$, the solution $u$ of $(4.5)$ is in $\Omega$.

In fact it is easy to observe that $u \equiv 0$ and hence $u \in \Omega$.

Claim 3. For $\lambda \in] 0,1]$ and $u$ a solution of (4.5) with $u(-n)<1$ and $u(n)>-1$, we have, $\forall t \in[0, n],-1<u(t)<\beta$ and, for all $t \in[-n, 0]$, $\alpha<u(t)<1$.

In case $u$ is constant, the result is trivial, so we can assume that $u$ is not constant. Let

$$
F_{+}(u)=\int_{0}^{u} f_{+}(s) d s \quad \text { and } \quad F_{-}(u)=\int_{0}^{u} f_{-}(s) d s .
$$

Observe that, if $u$ is a solution of $(4.5)$, then $f^{*}(\cdot, u(\cdot))$ is continuous in $[-n, n]$ and therefore $u \in \mathcal{C}^{3}([-n, n])$. Then

$$
\begin{aligned}
& \frac{d}{d t}\left[\lambda F_{+}(u(t))-u^{\prime}(t) u^{\prime \prime}(t)\right]=-u^{\prime \prime 2}(t)-p(t) u^{\prime 2}(t), \quad \text { for } t \in[0, n], \\
& \frac{d}{d t}\left[\lambda F_{-}(u(t))-u^{\prime}(t) u^{\prime \prime}(t)\right]=-u^{\prime \prime 2}(t)-p(t) u^{\prime 2}(t), \quad \text { for } t \in[-n, 0] .
\end{aligned}
$$

Since the solution is not constant and $F_{+}(u(0))=0=F_{-}(u(0))$, these inequalities yield

$$
F_{+}(u(n))<F_{-}(u(-n)) .
$$

Now the behaviour of $F_{+}$and $F_{-}$immediately implies $\left.u(n) \in\right]-1, \beta[$ and $u(-n) \in] \alpha, 1[$. Hence for all $t \in[0, n],-1<u(t)<\beta$ : otherwise suppose $\min _{0 \leq t \leq n} u(t)=u\left(t_{0}\right) \leq-1$. Then $\left.t_{0} \in\right] 0, n\left[, u^{\prime}\left(t_{0}\right)=0\right.$ and by the same argument $F_{+}\left(u\left(t_{0}\right)\right)<F_{-}(u(-n))$, a contradiction. A similar argument proves that $\max _{0 \leq t \leq n} u(t) \geq \beta$ cannot hold. We proceed in the same way to show that for all $0 \leq t \leq n$

$t \in[-n, 0], \alpha<u(t)<1$. 
Claim 4. For $\lambda \in] 0,1]$, there is no solution of (4.5) on $\partial \Omega$.

Otherwise, by Claim 3, we have a solution $u$ with either $u(-n)=1$ or $u(n)=-1$. If we have a solution $u$ with $u(-n)=1$, then, by (4.6) again, we have $F_{+}(u(n))<F_{-}(1)$ which contradicts the fact that $\min F_{+}=F_{-}(1)$. In the same way, if we have a solution $u$ with $u(n)=-1$, we find a similar contradiction.

Step 4. Conclusion of the proof. By standard results of Leray-Schauder degree theory, the equation (4.4) has a solution $u \in \Omega$ for $\lambda=1$ which is a solution of (4.2) and hence also a solution of (4.1) by Claim 3.

We have seen that, for all $n,\left\|u_{n}\right\|_{\mathcal{C}([-n, n])} \leq \max \{|\alpha|, \beta\}$. As in the proof of Lemma 3.3, we deduce

$$
\begin{aligned}
\left\|u^{\prime \prime}\right\|_{L^{2}(-n, n)}^{2} & \leq\left\|u^{\prime \prime}\right\|_{L^{2}(-n, n)}^{2}+\int_{-n}^{n} p(t) u^{\prime 2}(t) d t \\
& =F(u(-n))-F(u(n)) \leq 2 \max _{[\alpha, \beta]}|F(u)| .
\end{aligned}
$$

Define $v_{n}$ as the extension of $u_{n}$ to $\mathbb{R}$ such that, for all $t \leq-n, v_{n}(t)=u_{n}(-n)$ and, for all $t \geq n, v_{n}(t)=u_{n}(n)$. Then $v_{n} \in \mathcal{C}^{1}(\mathbb{R})$ and, as in Lemma 3.3, the Gagliardo-Nirenberg inequality yields the boundedness of

$$
\left\|v_{n}^{\prime}\right\|_{\mathcal{C}(\mathbb{R})}=\left\|u_{n}^{\prime}\right\|_{\mathcal{C}([-n, n])} .
$$

The proof then concludes as in Section 3.

REMARK 4.2. Theorem 4.1 extends to the non autonomous case [9, Theorem 0], [8] and [6, Theorem 5.2]. Even for the autonomous case our theorem improves the previous ones since we do not impose to the function $f$ to be $\mathcal{C}^{1}$ or Lipschitz continuous.

\section{Uniqueness of the kink solution}

In the following result we prove that, under condition (h4), the solutions of (1.5) have a unique zero.

Proposition 5.1. Suppose that $f$ and p satisfy conditions (f1), (p) and (h4). Then every solution $u \in \mathcal{C B}^{3}(\mathbb{R})$ of (1.5) has a unique zero $t_{0}$ and for all $t \in$ $\mathbb{R} \backslash\left\{t_{0}\right\}, u(t)\left(t-t_{0}\right)>0$.

Proof. Observe that, by Proposition 2.1, every solution $u \in \mathcal{C} B^{3}(\mathbb{R})$ of $(1.5)$ satisfies $u^{\prime}(+\infty)=u^{\prime \prime}(+\infty)=0$ and, by Proposition 2.3, for all $t \in \mathbb{R}, u(t) \in$ $[\alpha, \beta]$. Moreover, by assumptions, for all $x \in\left[\alpha, 0\left[, \int_{x}^{0} F(r) d r>0\right.\right.$ and, for all $x \in] 0, \beta], \int_{0}^{x} F(r) d r<0$.

Let us prove that, if $u$ is a solution of (1.5) such that $u\left(t_{0}\right)=0$, it satisfies $u^{\prime}\left(t_{0}\right)>0$ and hence a solution of (1.5) has a single zero. Assume by contradiction that $u^{\prime}\left(t_{0}\right) \leq 0$. 
Case 1. $u^{\prime \prime}\left(t_{0}\right) \leq 0$. As $u\left(t_{0}\right)=0, u^{\prime}\left(t_{0}\right) \leq 0, u^{\prime \prime}\left(t_{0}\right) \leq 0$ and $u^{\prime \prime \prime}\left(t_{0}\right)=$ $f(0)+p\left(t_{0}\right) u^{\prime}\left(t_{0}\right)<0$, we have that $u^{\prime}(t)$ is negative for values of $t$ close to $t_{0}$. Define $t_{1}=\sup \left\{t>t_{0} \mid\right.$ for all $\left.s \in\right] t_{0}, t\left[, u^{\prime}(s)<0\right\}$. As $u(+\infty)=1$, we have $t_{1}<+\infty, u^{\prime}\left(t_{1}\right)=0$ and $u^{\prime}(t)<0$ on $] t_{0}, t_{1}[$.

As $F(u(t))-u^{\prime}(t) u^{\prime \prime}(t)$ is nonincreasing along the solutions of (1.5), for $t \geq t_{0}$,

$$
F(u(t))-u^{\prime}(t) u^{\prime \prime}(t) \leq-u^{\prime}\left(t_{0}\right) u^{\prime \prime}\left(t_{0}\right) \leq 0 .
$$

Hence, we have

$$
0 \leq \int_{t_{0}}^{t_{1}}\left[F(u(t)) u^{\prime}(t)-u^{\prime 2}(t) u^{\prime \prime}(t)\right] d t=-\int_{u\left(t_{1}\right)}^{0} F(r) d r+\frac{u^{\prime 3}\left(t_{0}\right)}{3} .
$$

It follows that

$$
\int_{u\left(t_{1}\right)}^{0} F(r) d r \leq \frac{u^{\prime 3}\left(t_{0}\right)}{3} \leq 0,
$$

which contradicts $u\left(t_{1}\right) \in\left[\alpha, 0\left[\right.\right.$ and $\int_{u\left(t_{1}\right)}^{0} F(r) d r>0$.

Case 2. $u^{\prime \prime}\left(t_{0}\right)>0$. This case is similar to the previous one considering $u(t)$ for $t<t_{0}$.

Conclusion. We deduce from the two previous cases that $u^{\prime}\left(t_{0}\right)>0$ and hence $u$ has a unique zero.

In the following theorem we prove the uniqueness of solution for (1.5) under slightly stronger assumptions than in previous sections.

THEOREM 5.2. Suppose that $f$ and $p$ satisfy conditions (f1), (p), (h3) and (h4). Then, for every $t_{0} \in \mathbb{R}$, there exist $A>0$ and $B \in \mathbb{R}$ such that, for every solution $u \in \mathcal{C} B^{3}(\mathbb{R})$ of $(1.5)$ such that $u\left(t_{0}\right)=0$, we have $u^{\prime}\left(t_{0}\right)=A$ and $u^{\prime \prime}\left(t_{0}\right)=B$. Moreover the solution of (1.5) has a single zero. If moreover, $f$ is locally Lipschitz on $[\alpha, \beta]$, then, for every $t_{0} \in \mathbb{R}$, there exists at most one solution $u \in \mathcal{C B}^{3}(\mathbb{R})$ of $(1.5)$ such that $u\left(t_{0}\right)=0$. Moreover, $u$ is positive in ]$t_{0}, \infty[$, negative on $]-\infty, t_{0}[$ and

$$
u^{\prime}( \pm \infty)=u^{\prime \prime}( \pm \infty)=u^{\prime \prime \prime}( \pm \infty)=0 .
$$

Proof. Let $t_{0} \in \mathbb{R}$.

Step 1. There exists $A \in \mathbb{R}$ such that every solution $u$ of (1.5) such that $u\left(t_{0}\right)=0$ satisfies $u^{\prime}\left(t_{0}\right)=A$.

Otherwise, let $u_{1}$ and $u_{2}$ be two solutions with $u_{1}\left(t_{0}\right)=u_{2}\left(t_{0}\right)=0$ and $u_{1}^{\prime}\left(t_{0}\right)>u_{2}^{\prime}\left(t_{0}\right)$. Recall that, by Proposition 5.1, for every $t \neq t_{0}$, for $i=1,2$ we have $u_{i}(t)\left(t-t_{0}\right)>0$.

Let $w=u_{1}-u_{2}$ and observe that

$$
\begin{gathered}
w^{\prime \prime \prime}=f\left(u_{1}\right)-f\left(u_{2}\right)+p(t) w^{\prime}, \\
w\left(t_{0}\right)=0, \quad w^{\prime}\left(t_{0}\right)>0, \quad w(-\infty)=0, \quad w(+\infty)=0 .
\end{gathered}
$$


It follows that for $t$ close to $t_{0}, w^{\prime}(t)>0$ and since $w\left(t_{0}\right)=w(-\infty)=w(+\infty)=$ 0 , there exists $t_{1}<t_{0}<t_{2}$ such that $w^{\prime}(t)>0$ on $] t_{1}, t_{2}\left[\right.$ and $w^{\prime}\left(t_{1}\right)=w^{\prime}\left(t_{2}\right)=0$. Next as $f$ is nondecreasing on $\mathbb{R}^{+}$and nonincreasing on $\mathbb{R}^{-}$and, for every $t \neq t_{0}$, for $i=1,2$ we have $u_{i}(t)\left(t-t_{0}\right)>0$, we have that $z=w^{\prime}$ satisfies

$$
\begin{gathered}
\left.z^{\prime \prime}=f\left(u_{1}\right)-f\left(u_{2}\right)+p(t) z>0, \quad \text { on }\right] t_{1}, t_{2}[, \\
z\left(t_{1}\right)=z\left(t_{2}\right)=0, \quad z\left(t_{0}\right)>0,
\end{gathered}
$$

which contradicts the maximum principle.

Step 2. There exists $B \in \mathbb{R}$ such that every solution $u$ of (1.5) such that $u\left(t_{0}\right)=0$ satisfies $u^{\prime \prime}\left(t_{0}\right)=B$.

Otherwise, let $u_{1}$ and $u_{2}$ be two solutions with $u_{1}\left(t_{0}\right)=u_{2}\left(t_{0}\right)=0$ and $u_{1}^{\prime \prime}\left(t_{0}\right)>u_{2}^{\prime \prime}\left(t_{0}\right)$. By Step 1 , we have $u_{1}^{\prime}\left(t_{0}\right)=u_{2}^{\prime}\left(t_{0}\right)$. As in Step 1 , we observe that there exists $t_{2}>t_{0}$ such that $w=u_{1}-u_{2}$ satisfies

$$
\begin{gathered}
\left.w^{\prime \prime \prime}=f\left(u_{1}\right)-f\left(u_{2}\right)+p(t) w^{\prime}, \quad \text { on }\right] t_{0}, t_{2}[, \\
w\left(t_{0}\right)=0, \quad w^{\prime}\left(t_{0}\right)=0, \quad w^{\prime \prime}\left(t_{0}\right)>0, \quad w^{\prime}\left(t_{2}\right)=0,
\end{gathered}
$$

and hence $z=w^{\prime}$ satisfies

$$
\begin{gathered}
\left.z^{\prime \prime}=f\left(u_{1}\right)-f\left(u_{2}\right)+p(t) z>0, \quad \text { on }\right] t_{0}, t_{2}[, \\
z\left(t_{0}\right)=z\left(t_{2}\right)=0, \quad z^{\prime}\left(t_{0}\right)>0,
\end{gathered}
$$

which contradicts the Hopf maximum principle.

Combining Theorems 4.1 and 5.2 we obtain the following result in the particular case where $p$ is a constant.

TheOREM 5.2. Suppose that $f: \mathbb{R} \rightarrow \mathbb{R}$ is locally Lipschitz on $[\alpha, \beta]$ and satisfies (f1), (h3) and (h4). In addition assume $p$ is a nonnegative constant. Then (1.5) has a unique (up to translations) solution $u \in \mathcal{C B}^{3}(\mathbb{R})$. Moreover, $u$ has a unique simple zero and

$$
u^{\prime}( \pm \infty)=u^{\prime \prime}( \pm \infty)=u^{\prime \prime \prime}( \pm \infty)=0 .
$$

In the symmetric case, we obtain the following result.

TheOREm 5.4. Suppose that $f: \mathbb{R} \rightarrow \mathbb{R}$ is locally Lipschitz on $[\alpha, \beta]$ and satisfies conditions (f1), (p), (h3) and (s). Then there exists at most one solution $u \in \mathcal{C B}^{3}(\mathbb{R})$ of $(1.5)$ such that $u(0)=0$.

If $\left(s^{\prime}\right)$ is satisfied too, then the problem (1.5) has a unique solution $u$ such that $u(0)=0$. Moreover, $u$ is odd, positive on $[0,+\infty[$ and satisfies

$$
u^{\prime}( \pm \infty)=u^{\prime \prime}( \pm \infty)=u^{\prime \prime \prime}( \pm \infty)=0 .
$$


ProOF. We just have to prove that (h4) is satisfied. In that case, we conclude by Theorems 4.1 and 5.2. Let $u_{1}$ be the positive zero of $F$ and assume $\int_{0}^{\beta} F(s) d s \geq 0$. As $F$ is convex on $[0, \beta]$ and $-F(1)=F(\beta)>0$, we have $1<(1+\beta) / 2<u_{1}<\beta$. Hence, we obtain the contradiction

$$
\int_{u_{1}}^{\beta} F(r) d r \geq\left|\int_{0}^{u_{1}} F(r) d r\right|>\left|\int_{1}^{u_{1}} F(r) d r\right|>|F(1)| \frac{\beta-1}{4}>\int_{u_{1}}^{\beta} F(r) d r,
$$

which proves that $\int_{0}^{\beta} F(s) d s>0$. The proof that $\int_{\alpha}^{0} F(s) d s<0$ is similar.

REMARK 5.5. Theorem 5.4 extends the uniqueness result in [12, Theorem 3.8] to the nonautonomous case.

As an immediate consequence of the previous results we have the following one for the model problem.

Corollary 5.6. Consider the problem

$$
\lambda u^{\prime \prime \prime}=u^{2}-1, \quad u(-\infty)=-1, \quad u(+\infty)=1 .
$$

Then we have:

(a) For $\lambda>0$ there exists a unique solution $u \in \mathcal{C}^{3}(\mathbb{R})$ of (5.1) (up to translations). Moreover, u has a unique simple zero, is odd around it, and

$$
u^{\prime}( \pm \infty)=u^{\prime \prime}( \pm \infty)=u^{\prime \prime \prime}( \pm \infty)=0 .
$$

(b) For $\lambda<0$ problem (5.1) has no solution.

\section{REFERENCES}

[1] A. Bressan, Hyperbolic Systems of Conservation Laws. The One-Dimensional Cauchy Problem, Oxford Lecture Series in Mathematics and its Applications, vol. 20, Oxford University Press, 2000.

[2] D. Bonheure and L. Sanchez, Heteroclinic Orbits for Some Classes of Second and Fourth Order Differential Equations, Handbook of Differential Equations: Ordinary Differential Equations, vol. . 3 (A. Cañada, P. Drabek, A. Fonda, eds.), Elsevier, 2006.

[3] C. Conley, Isolated Invariant Sets and the Morse Index, C.B.M.S., vol. 38, Amer. Math. Soc., Providence, 1978.

[4] I.M. GEL'FAnd, Some problems in the theory of quasi-linear equations, Uspehi Mat. Nauk 14 (1959), 87-158.

[5] N. Kopell and L.N. Howard, Bifurcations and trajectories joining critical points, Adv. Math. 18 (1975), 306-358.

[6] V. Manukian And S. SChecter, Travelling waves for a thin liquid film with surfactant on an inclined plane, Nonlinearity 22 (2009), 85-122.

[7] Ch.K. McCord, Uniqueness of connecting orbits in the equation $Y^{(3)}=Y^{2}-1$, J. Math. Anal. Appl. 114 (1986), 584-592.

[8] M.S. Mock, On fourth-order dissipation and single conservation laws, Comm. Pure Appl. Math. 29 (1976), 383-388. 
[9] The half-line boundary value problem for $u_{x x x}=f(u)$, J. Differential Equations 32 (1979), 258-273.

[10] L. Nirenberg, On elliptic partial differential equations, Ann. Scuola Norm. Sup. Pisa 13 (1959), 115-162.

[11] J. Smoller, Shock Waves and Reaction-Diffusion Equations, Springer-Verlag, New York, 1983.

[12] J.F. Toland, Existence and uniqueness of heteroclinic orbits for the equation $\lambda u^{\prime \prime \prime}+$ $u^{\prime}=f(u)$, Proc. Royal Soc. Edinburgh 109A (1988), 23-36.

[13] W. Walter, Ordinary Differential Equations, Springer-Verlag, New York, 1998.

Manuscript received December 29, 2011

Denis Bonheure

Département de Mathématique

Université libre de Bruxelles

CP 214 Boulevard du Triomphe

1050 Brussels, BELGIUM

E-mail address: dbonheur@ulb.ac.be

José Ángel Cid

Departamento de Matemáticas

Universidade de Vigo

Pabellón 3

Campus de Ourense

32004, Ourense, SPAIN

E-mail address: angelcid@uvigo.es

Colette De Coster

Université de Valenciennes

et du Hainaut Cambrésis

LAMAV, FR CNRS 2956

Institut des Sciences et Techniques de Valenciennes

F-59313 Valenciennes, Cedex 9, FRANCE

E-mail address: Colette.DeCoster@univ-valenciennes.fr

LuÍS SANCHEZ

CMAF - Faculdade de Ciências

da Universidade de Lisboa

Avenida Professor Gama Pinto 2

1649-003 Lisboa, PORTUGAL

E-mail address: sanchez@ptmat.fc.ul.pt 